\title{
Análise de séries temporais dos preços da soja utilizando o método Gráfico de Recorrência
}

Analysis of time series of the soybean prices using the Recurrence Plot method

Análisis de series temporales de los precios de la soja mediante el método Gráfico de Recurrencia

Recebido: 03/06/2021 | Revisado: 13/06/2021 | Aceito: 17/06/2021 | Publicado: 02/07/2021

Evellyn Cabral

ORCID: https://orcid.org/0000-0002-6132-408X Universidade Federal Rural de Pernambuco, Brasil E-mail: cabral.eveellyn@gmail.com

Leika Irabele Tenório de Santana

ORCID: https://orcid.org/0000-0002-5516-5887 Universidade Federal Rural de Pernambuco, Brasil E-mail: leikatenorio10@gmail.com

Lidiane da Silva Araújo

ORCID: https://orcid.org/0000-0003-3111-7925 Universidade Federal Rural de Pernambuco, Brasil E-mail: lidiane_sac@hotmail.com

Tatijana Stosic

ORCID: https://orcid.org/0000-0002-5691-945X Universidade Federal Rural de Pernambuco, Brasil E-mail: tastosic@gmail.com

\begin{abstract}
Resumo
O Agronegócio é a atividade que mais movimenta a economia mundial, não só pela produção de alimentos destinados ao consumo interno e à exportação, como também por sustentar outros setores econômicos. O Brasil é o país que mais se destaca nesse tipo de atividade que vem crescendo desde os últimos 40 anos. A atividade brasileira do Agronegócio voltada ao mercado de exportação concentra-se, em sua maior parte, à produção de commodities agrícolas. Entre elas, uma que se tem destacado é a soja em grãos, que em 2020 levou o Brasil a se tornar seu maior produtor e o mantém no ranking de maior exportador mundial. Tendo em vista a grande importância econômica desse grão para o País e a importância de se investigarem os possíveis impactos que crises financeiras podem causar em seu preço, este trabalho buscou apresentar um estudo sobre os possíveis efeitos da crise financeira de 2008 (crise do Subprime) no preço da soja. Foram então analisados os dados diários do preço da soja registrados entre 29/07/1997 e 30/12/2020 pelo CEPEA-ESALQ/USP. Utilizou-se o método Gráfico de Recorrência e a Análise de Quantificação de Recorrência. Os resultados mostraram que a crise do Subprime influenciou a dinâmica de preços da soja de forma que ela passou a apresentar maior grau de previsibilidade no período pós-crise, sugerindo que o mercado dessa commodity era mais eficiente antes da crise e que o mercado externo da soja é mais eficiente que seu mercado interno.
\end{abstract}

Palavras-chave: Commodities agrícolas; Crise do Subprime; Gráfico de recorrência; Análise de quantificação de recorrência.

\begin{abstract}
Agribusiness is the activity that most moves the world economy, not only for the production of food for domestic consumption and export, but also for sustaining other economic sectors. Brazil is the country that stands out the most in this type of activity that has been growing since the last 40 years. The brazilian Agribusiness activity focused on the export market is concentrated, for the most part, on the production of agricultural commodities. Among them, one that has stood out is soybeans, which in 2020 led Brazil to become its largest producer and keeps the country in the ranking of the world's largest exporter. In view of the great economic importance of this grain for the country and the importance of investigating the possible impacts that financial crises may have on its price, this paper sought to present a study on the possible effects of the 2008 financial crisis (Subprime crisis) in the price of soybean. The daily soybean price data recorded between 07/29/1997 and 12/30/2020 by CEPEA-ESALQ/USP were then analyzed. The Recurrence Plot method and the Recurrence Quantification Analysis were used. The results showed that the Subprime crisis influenced the soybean price dynamics in a way that it started to show a higher degree of predictability in the post-crisis period, suggesting that the market of this commodity was more efficient before the crisis and that the foreign market of soy is more efficient than its domestic one.
\end{abstract}

Keywords: Agricultural commodities; Subprime crises; Recurrence plot; Recurrence quantification analysis. 


\begin{abstract}
Resumen
La agroindustria es la actividad que más mueve la economía mundial, no solo para la producción de alimentos para consumo interno y exportación, sino también para sostener otros sectores económicos. Brasil es el país que más se destaca en este tipo de actividad que viene creciendo durante los últimos 40 años. La actividad agroindustrial brasileña centrada en el mercado de exportación se concentra, en su mayor parte, en la producción de commodities agrícolas. Entre ellas, una que ha destacado es la soja en granos, que en 2020 llevó el Brasil a convertirse en su mayor productor y lo mantiene en el ranking de mayor exportador mundial. Dada la gran importancia económica de este grano para el país y la importancia de investigar posibles impactos que crisis financieras puedan tener en su precio, este trabajo buscó presentar un estudio sobre los posibles efectos de la crisis financiera de 2008 (crisis Subprime) en el precio de soja. Para esto, se analizaron los datos de precios diarios de soja registrados entre 29/07/1997 y 30/12/2020 por CEPEA-ESALQ/USP. Se utilizó el método Gráfico de Recurrencia y la Análisis de Cuantificación de Recurrencia. Los resultados mostraron que la crisis Subprime influyó en la dinámica del precio de la soja de forma que los datos mostraron mayor grado de previsibilidad en el período posterior a la crisis, sugiriendo que el mercado de esta commodity era más eficiente antes de la crisis y que el mercado externo de soja es más eficiente que su mercado doméstico.
\end{abstract}

Palabras clave: Commodities agrícolas; Crisis Subprime; Gráfico de recurrencia; Análisis de cuantificación de recurrencia.

\title{
1. Introdução
}

De acordo com os pesquisadores Davis e Goldberg (1957), o Agronegócio se entende como a soma total das operações de produção e distribuição de suprimentos agrícolas, dos processos na unidade de produção, do armazenamento, do processamento e da distribuição dos produtos agrícolas e dos itens produzidos por meio deles. Essa atividade de grande importância mundial se tornou a principal fonte de desenvolvimento do Brasil, dada a sua forte contribuição para o crescimento econômico, a geração de empregos e, consequentemente, o bem-estar social que ela promove. O Agronegócio brasileiro é hoje capaz de produzir diversos tipos de matérias-primas e oferecer subsídios para o setor têxtil, de celulose, biocombustíveis, medicamentos, entre outros. De acordo com o Ministério da Agricultura (Salomão, 2020), a atividade rendeu ao País US\$ 96,79 bilhões no ano de 2019, o que corresponde a 21,4\% do PIB brasileiro, segundo o Centro de Estudos Avançados em Economia Aplicada - CEPEA (CEPEA/ESALQ/USP, 2021) em parceria com a Confederação de Agricultura e Pecuária - CNA (2021) e a Fundação de Estudos Agrários Luiz de Queiroz - FEALQ (2021). Em 2020, ano em que todos os países sofreram com os efeitos negativos traduzidos pela pandemia do coronavírus, o Agronegócio brasileiro se manteve em crescimento graças à atividade de exportação de suas principais commodities agrícolas.

O Brasil é um grande produtor e exportador de commodities. Ele registrou em 2020 volumes recordes de exportação de suas principais commodities, segundo o Ministério da Economia (SNA, 2021). Entre elas, a mais exportada foi a soja, que, segundo a Associação de Comércio Exterior do Brasil, foi considerada pelo sexto ano seguido a principal commodity agrícola que o País exporta, e com estimativas de que continue a ser em 2021.

A produção de soja vem crescendo contínua e expressivamente desde a sua expansão em 1970. Isso vem gerando impactos positivos em diversos setores por estar ligada a um vasto número de agentes e organizações, como social, econômico, ambiental, político e principalmente tecnológico, já que continuamente se desenvolvem mais métodos genéticos e mecânicos para que o manejo dessa commodity alcance produtividade cada vez maior e mais sustentável (Gouveia et al., 2020; Mauad et al., 2010). A importância econômica da soja se destaca não somente na exportação do grão, mas também na produção de farelo (também para exportação), óleo e biodiesel (Picolli, 2018). Além disso, a soja é usada como insumo junto ao milho para a produção de ração animal (Gouveia et al., 2020).

Dito isso, é de suma importância estudar o comportamento da dinâmica de preços dessa commodity agrícola frente a uma crise financeira como a de 2008 (crise do Subprime), que afetou a economia mundial. Uma das consequências da crise foi o aumento dos preços de commodities alimentares (por isso também é conhecida como a crise alimentar - food crises), que resultou na insegurança alimentar e movimentos sociais em países em desenvolvimento (Ivanic et al., 2012). 
Devido à importância da soja na economia do Brasil (seu maior exportador), e conhecendo a intensidade com que a crise de 2008 afetou a economia mundial, o objetivo deste trabalho é investigar os possíveis impactos que ela provocou no preço dessa commodity agrícola brasileira. Para isso, foram analisados os dados diários de preços da soja (em moeda brasileira - BRL e dólar americano - USD) coletados pelo CEPEA - ESALQ/USP entre os anos de 1997 e 2020. Os métodos utilizados foram o Gráfico de Recorrência e a Análise de Quantificação de Recorrência, idealizados para o estudo de séries temporais não lineares com base nas recorrências dos estados de sistemas dinâmicos no espaço de fase (Marwan et al., 2007; Zbilut \& Webber Jr, 1992).

\section{Metodologia}

\subsection{Descrição dos dados}

São analisados os dados de preços diários da soja brasileira em BRL e USD registrados entre 29/07/1997 e 30/12/2020 pelo Centro de Estudos Avançados em Economia Aplicada / Escola Superior de Agricultura Luiz de Queiroz / Universidade de São Paulo (CEPEA/ESALQ/USP, 2021). Além da série temporal com os dados de todo o período, são analisadas as séries correspondentes ao período pré-crise, de 29/07/1997 a 13/07/2007, e pós-crise, de 16/07/2007 a 30/12/2020 (Kristoufek et al., 2012). O preço da soja possui o indicador CEPEA/ESALQ - PARANÁ, com valor em reais por saca de 60 $\mathrm{kg}$ (R\$/saca) (CEPEA/ESALQ/USP, 2021). A Figura 1 ilustra as séries temporais originais de preços da soja em todo o período (a) e nos períodos pré-crise (b) e pós-crise (c).

Figura 1: Séries temporais originais de preços da soja em moeda brasileira (BRL) e dólar americano (USD) em todo o período (a), no período pré-crise (b) e no pós-crise (c).
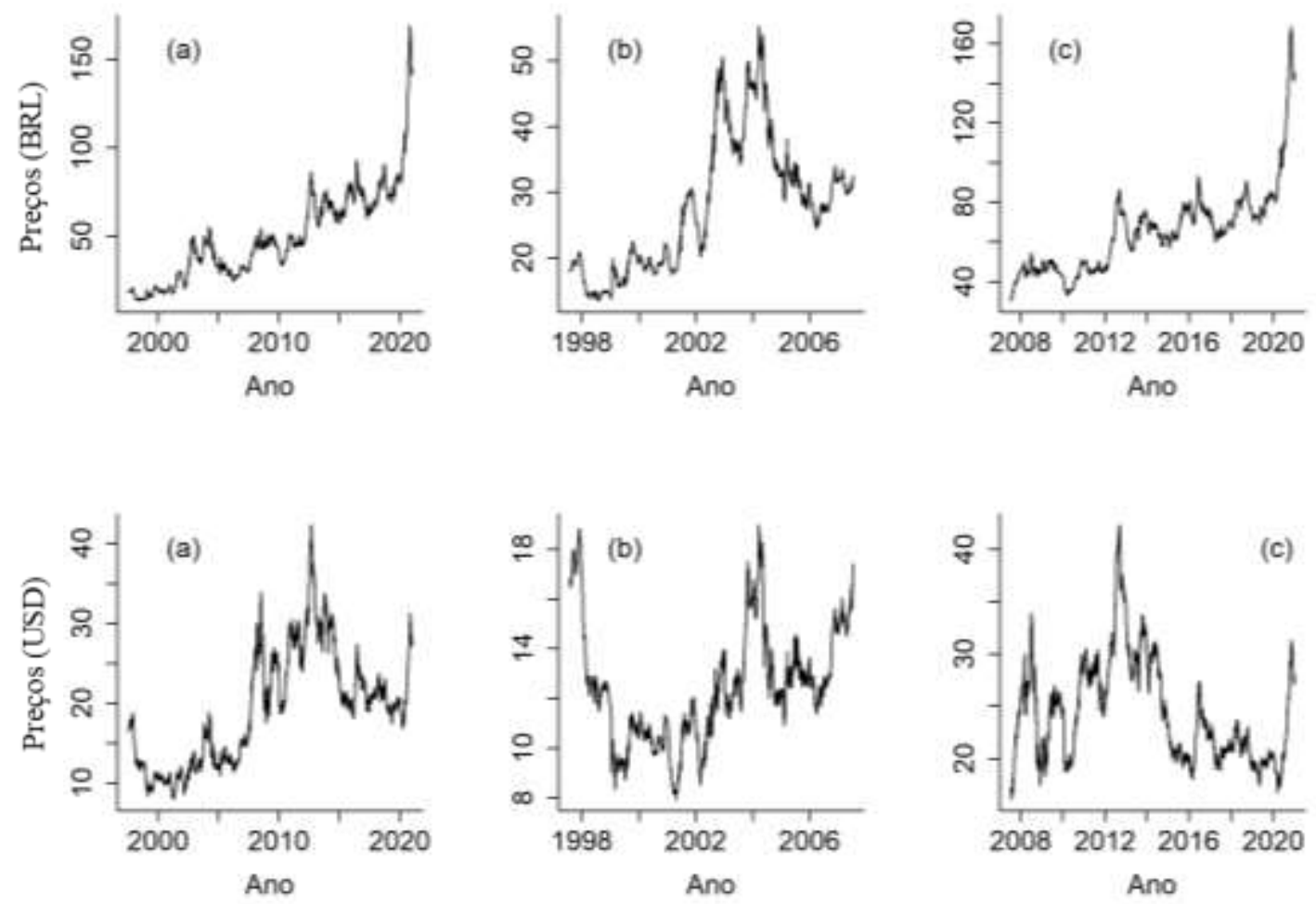

Fonte: Elaborado pelos autores a partir dos dados do CEPEA (2021). 


\subsection{O método Gráfico de Recorrência}

Eckmann et al. (1987) desenvolveram o método Gráfico de Recorrência (Recurrence Plot - RP) que serve para visualizar as recorrências de sistemas dinâmicos. Este método foi amplamente utilizado em análise de dados em Fisiologia (Desai et al., 2016), Geofísica (Donner et al., 2019), Climatologia (Shu et al., 2021), Hidrologia (de Santana et al., 2020), Ecologia (Barros et al., 2021) e Finanças (Bastos \& Caiado, 2011).

O algoritmo de construção do RP é assim descrito: para uma série temporal escalar $\left\{u_{i}\right\}$ constroem-se os vetores $\vec{x}_{i}=\left(u_{i}, u_{i+\tau}, \ldots, u_{i+(m-1) \tau}\right)$, em que $m$ é a dimensão de imersão e $\tau$ é o tempo de retardo (Takens, 1981). Os métodos mais utilizados para a obtenção dos valores dos parâmetros $m$ e $\tau$ são o método dos vizinhos falsos mais próximos e a função de informação mútua, respectivamente (Cao, 1997; Kantz \& Schreiber, 2004). O RP é matematicamente representado pela matriz quadrada $N \times N$ dada por

$$
R_{i, j}=\Theta\left(\varepsilon-\left\|\vec{x}_{i}-\vec{x}_{j}\right\|\right), \quad i, j=1, \ldots, N
$$

onde $\vec{x}_{i}$ e $\vec{x}_{j}$ são os vetores (estados) no espaço de fase, $\varepsilon$ é o raio de vizinhança, $\Theta$ é a função de Heaviside e $\|\cdot\|$ é a norma (euclidiana, por exemplo). Se os estados $\vec{x}_{i}$ e $\vec{x}_{j}$ são similares (recorrentes), $R_{i, j}=1$ e um ponto preto na coordenada $(i, j)$ é então plotado no RP; caso contrário, $R_{i, j}=0$ e um ponto branco é plotado no RP. Por definição, $\left.R_{i, i} \equiv 1\right|_{i=1} ^{N}$, gerando uma linha diagonal principal chamada de linha de identidade (line of identity - LOI); $R_{i, j}=R_{j, i}$ e o RP será simétrico a LOI (Eckmann et al., 1987; Marwan et al., 2007).

Os padrões dos RPs são relacionados à evolução temporal do sistema dinâmico. Esses padrões são divididos em duas categorias: padrões de larga escala (tipologia) e padrões de pequena escala (textura). Padrões de larga escala são classificados em homogêneo (geralmente associado a processo aleatório), periódico (caracterizado por linhas diagonais paralelas a LOI), deriva (ausência de pontos recorrentes no canto superior esquerdo e no canto inferior direito, gerada por processo não estacionário que contém uma tendência), e descontínuo (possuem faixas brancas, indicando mudanças abruptas, como eventos extremos). Os padrões da pequena escala são pontos e linhas. Os pontos representam estados recorrentes (pontos isolados são características das recorrências raras, típicas dos processos aleatórios) e as linhas podem ser diagonais (ocorrem quando um segmento de trajetória evolui paralelamente a outro segmento, como no caso dos sistemas oscilantes) e verticais/horizontais (ocorrem quando o estado do sistema não muda ou muda lentamente) (Eckmann et al., 1987; Marwan et al., 2007).

\subsection{Análise de Quantificação de Recorrência}

As medidas realizadas com a Análise de Quantificação de Recorrência (Recurrence Quantification Analysis - RQA) foram desenvolvidas por Zbilut e Webber Jr (1992), e Marwan et al. (2002). Elas são obtidas com base nos padrões de pequena escala: pontos, estruturas diagonais e verticais. As medidas baseadas em pontos e estruturas diagonais são: taxa de recorrência - REC (mede a densidade dos pontos de recorrências no RP), determinismo - DET (mede o percentual de pontos de recorrências que formam as estruturas diagonais), comprimento médio das linhas diagonais - $L$ (considerado como tempo médio de previsão do sistema), comprimento máximo da linha diagonal - $L_{\max }$ (tamanho da linha diagonal mais longa, excluindo $L O I$ ) e divergência - DIV (que é o inverso de $L_{\max }$ e indica o quão rápido os segmentos de trajetórias divergem no espaço da fase). As medidas baseadas nas estruturas verticais são: laminariedade - LAM (mede o percentual de pontos de recorrências que formam as estruturas verticais) e tempo de aprisionamento, trapping time - TT (comprimento médio das 
linhas verticais que fornece o tempo médio em que o sistema permanece num estado laminar, estado que não muda com o tempo).

\section{Resultados e Discussão}

As séries temporais de preços da soja considerando o período completo e os períodos pré-crise e pós-crise foram normalizadas no intervalo entre 0 e 1 , segundo a expressão

$$
P=\frac{x-\min (x)}{\max (x)-\min (x)}
$$

onde $x$ denota a série original, e $\max (x)$ e $\min (x)$ são os valores de máximo e mínimo de $x$, respectivamente (Bastos \& Caiado, 2010). A Figura 2 ilustra as séries normalizadas dos preços de soja no período completo (a), no pré-crise (b) e no póscrise (c). Todas as análises foram realizadas com uso do software R Core Team (2020).

Figura 2: Séries normalizadas de preços da soja em moeda brasileira (BRL) e dólar americano (USD) no período completo (a), no pré-crise (b) e no pós-crise (c).
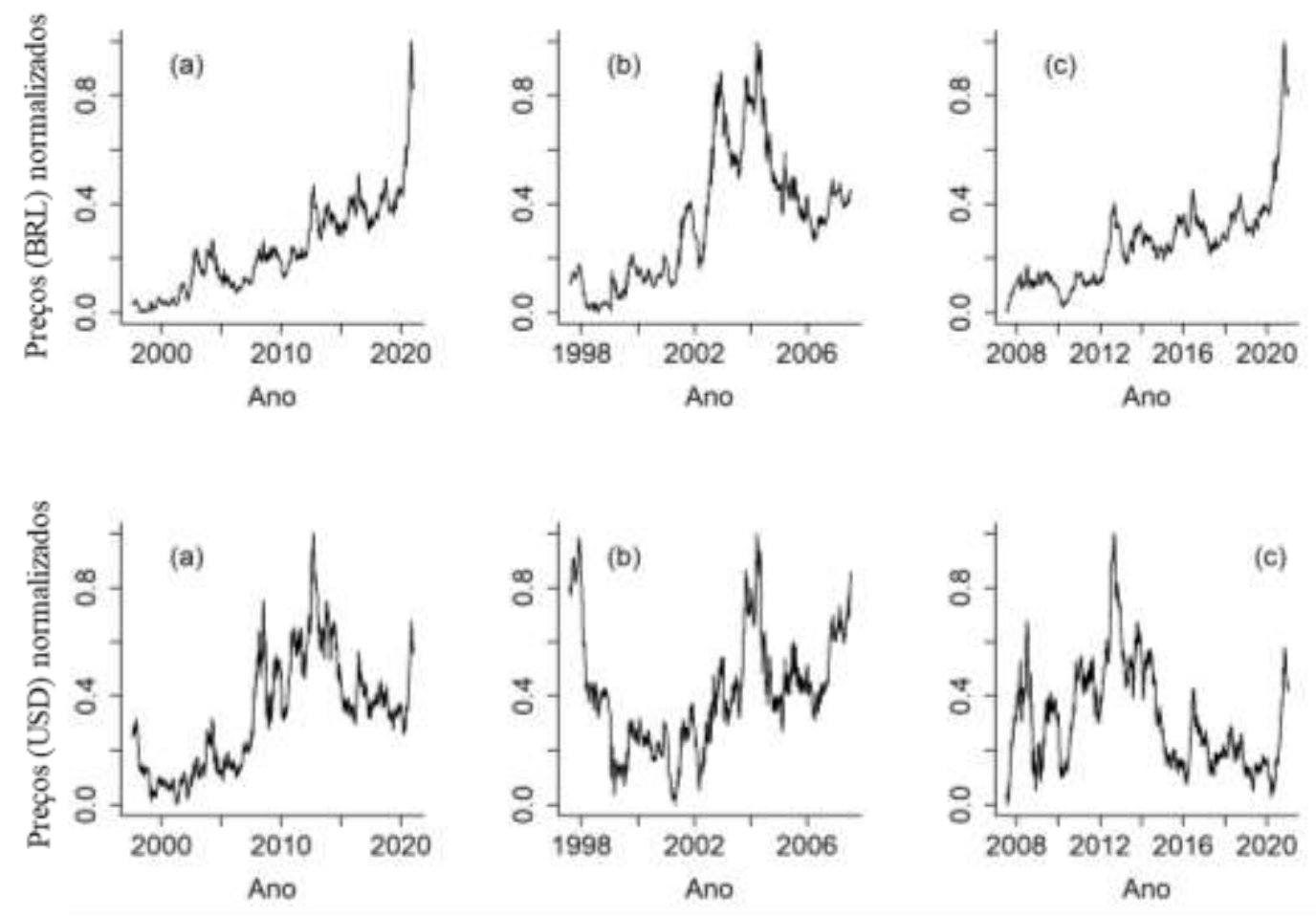

Fonte: Autores (2021).

Adotou-se $\tau=1$, valor de tempo de retardo usualmente utilizado para séries temporais financeiras (Bastos \& Caiado, 2010; Yao \& Lin, 2017; Zbilut, 2005). O valor estimado para a dimensão de imersão foi $m=9$, obtido pelo método dos falsos vizinhos mais próximos (Cao, 1997; Kantz \& Schreiber, 2004). O valor do limiar $\varepsilon$ foi escolhido para gerar a taxa de recorrência REC entre $2 \%$ e $5 \%$ (Webber \& Zbilut, 2005). Os valores do parâmetro $\varepsilon$ obtidos para os RPs encontram-se listados na Tabela 1. 
Tabela 1: Valores do limiar $\varepsilon$ obtidos para cada série temporal.

\begin{tabular}{ccccc}
\hline & Moeda & Período completo & Pré-crise & Pós-crise \\
\hline \multirow{2}{*}{ Limiar $\varepsilon$} & BRL & 0,014 & 0,022 & 0,016 \\
\cline { 2 - 5 } & USD & 0,024 & 0,034 & 0,028 \\
\hline
\end{tabular}

Fonte: Autores (2021).

Da Tabela 1 observa-se que os valores do limiar $\varepsilon$ são distintos. Para aplicação da Análise de Quantificação de Recorrência, utiliza-se o valor médio (Bastos \& Caiado, 2010); isto é, $\varepsilon=0,017$ para preços em real e $\varepsilon=0,029$ para preços em dólar.

Os Gráficos de Recorrência relacionados aos preços da soja em moeda brasileira no período completo (a), no pré-crise (b) e no pós-crise (c) são mostrados na Figura 3. Observa-se a existência de bandas brancas no RP para o período pré-crise entre os anos de 2002 e 2007 e para o pós-crise entre 2011 e 2020. Essas são características de sistemas descontínuos e indicam que houve variações abruptas temporais (Marwan et al., 2007), o que está de acordo com a Figura 2. Além disso, verifica-se que as oscilações no preço não ocorrem de forma aleatória, já que não há a presença apenas de pontos isolados. Os RPs apresentam ainda linhas verticais e horizontais, caracterizando a existência de estados laminares, indicando que o sistema vai se alterar lentamente ou não se alterar; e pequenas linhas diagonais, paralelas a LOI, que representam um comportamento caótico não estacionário (Marwan et al., 2007).

Figura 3: Gráficos de recorrência de preços da soja em moeda brasileira (BRL) para o período completo (a), o pré-crise (b) e o pós-crise (c).

(a)

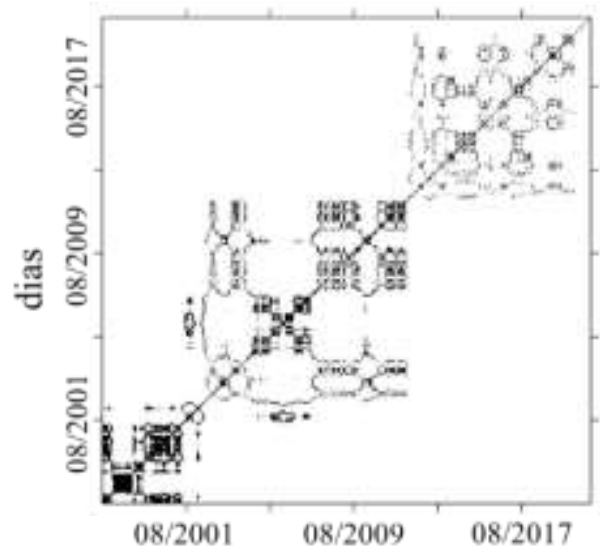

(b)

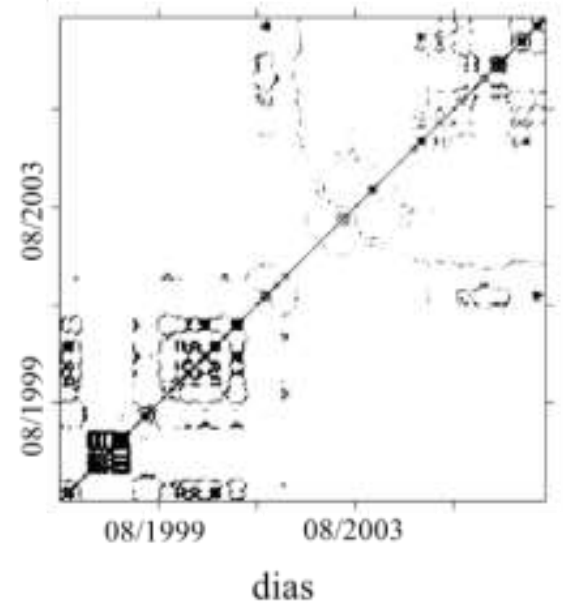

Fonte: Autores (2021). (c)

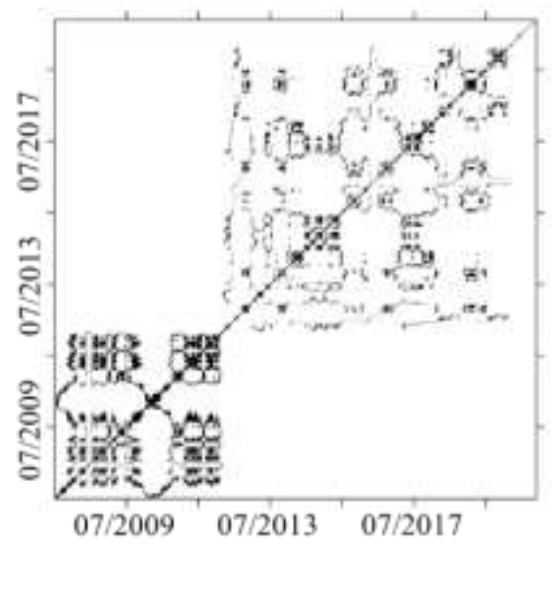

Os Gráficos de Recorrência relacionados ao preço da soja em dólar americano no período completo (a), no pré-crise (b) e no pós-crise (c) são mostrados na Figura 3. Observa-se a existência de bandas brancas no RP entre os anos de 1998 e 2001 para o período pré-crise e entre os anos de 2010 e 2014 para o período pós-crise, indicando os períodos quando ocorreram variações temporais abruptas (Marwan et al., 2007), o que pode ser confirmado na Figura 2. A presença das linhas verticais e horizontais indica a existência de estados laminares (o sistema varia lentamente), e linhas diagonais curtas paralelas a LOI indicando um comportamento caótico não estacionário (Marwan et al., 2007). 
Figura 4: Gráficos de recorrência de preços da soja em dólar americano (USD) para o período completo (a), o pré-crise (b) e o pós-crise (c).

(a)

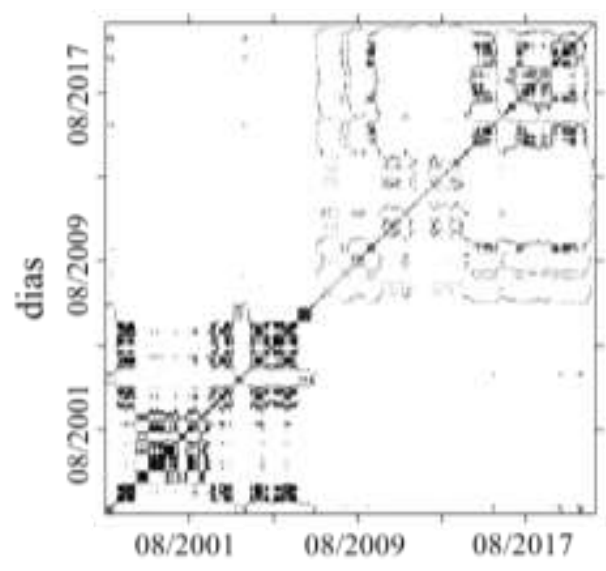

(b)

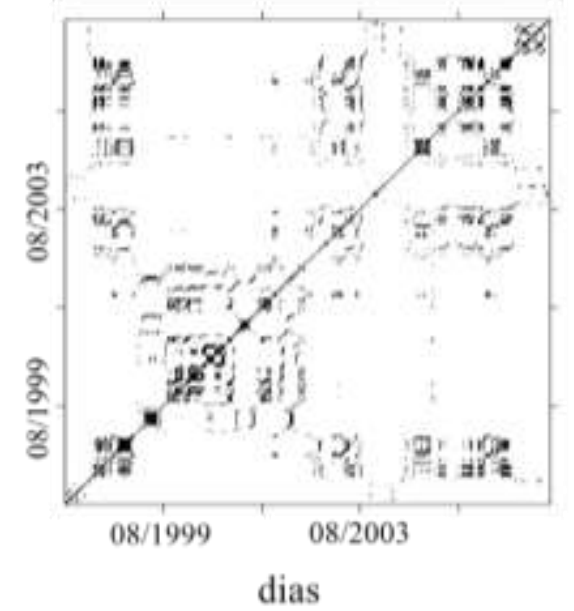

(c)

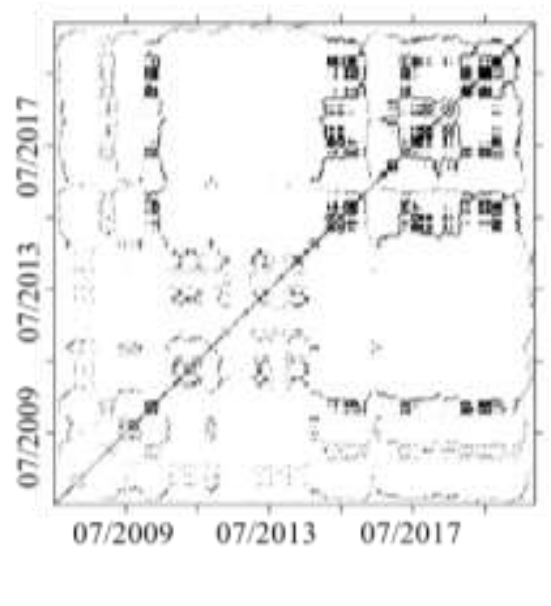

Fonte: Autores (2021).

As medidas de quantificação da RQA são apresentadas na Tabela 2. Observa-se que para ambas as séries de preços (em BRL e USD) os valores de todos os índices aumentaram depois da crise, indicando uma maior previsibilidade dos preços. O aumento dos índices indica: i) REC - maior densidade de pontos recorrentes; ii) DET - maior proporção de pontos recorrentes formando linhas diagonais ou mais padrões repetitivos ou determinísticos na dinâmica; iii) $L$ - maior comprimento médio das linhas diagonais, maior tempo de previsão do sistema; iv) $L_{\max }$ - maior comprimento de linha diagonal (excluindo LOI), menos caótico (mais estável) o processo; v) TT - maior comprimento médio de linhas verticais, maior tempo de permanência em estados que mudam lentamente. Em suma, esses resultados indicam que a crise do Subprime influenciou o mercado brasileiro da soja, sugerindo que ele tenha se tornado menos eficiente depois da crise. Comparando os índices de RQA entre as séries de preços em BRL e USD, observa se que para ambos os períodos os índices são menores para as séries de preços em USD, indicando que o mercado externo da soja é mais eficiente que seu mercado interno.

Recentemente o RP e a RQA foram aplicados em análise de preços de soja milho e frango, e constatou-se que os preços dessas commodities evoluem de maneira semelhante, e que os preços de soja e milho são mais sincronizados entre si do que entre cada um deles e os de frango (de Santana et al., 2020). Os resultados deste trabalho providenciam informações sobre a variação dos preços de soja nos mercados externo e interno e a influência da crise financeira 2008. 
Tabela 2: Medidas de quantificação de recorrência para os preços de soja em BRL e USD no período completo e nos períodos pré-crise e pós-crise.

\begin{tabular}{|c|c|c|c|c|c|c|}
\hline & \multicolumn{3}{|c|}{ BRL } & \multicolumn{3}{|c|}{ USD } \\
\hline Medida & Completo & Pré-crise & Pós-crise & Completo & Pré-crise & Pós-crise \\
\hline$R E C$ & 0,046 & 0,016 & 0,042 & 0,047 & 0,012 & 0,027 \\
\hline$D E T$ & 0,995 & 0,985 & 0,990 & 0,995 & 0,970 & 0,981 \\
\hline$L$ & 19,183 & 10,639 & 12,081 & 17,715 & 7,058 & 9,443 \\
\hline$L_{\max }$ & 2275 & 356 & 1235 & 1761 & 149 & 351 \\
\hline$L A M$ & 0,987 & 0,957 & 0,973 & 0,974 & 0,876 & 0,918 \\
\hline$T T$ & 17,751 & 9,096 & 10,302 & 14,355 & 4,892 & 6,567 \\
\hline
\end{tabular}

Fonte: Autores (2021).

\section{Considerações Finais}

Neste trabalho investigaram-se os efeitos da crise do Subprime (2008) nas séries diárias de preços da soja utilizando o método de Gráfico de Recorrência (Recurrence Plot - RP) e a Análise de Quantificação de Recorrência (Recurrence Quantification Analysis - RQA), com o auxílio do software R Core Team. Analisaram-se os preços em real e em dólar americano para o período de 29/07/1997 a 30/12/2020. Os resultados mostraram que depois da crise a dinâmica de preços apresentou maior grau de previsibilidade e laminaridade mais forte (presença de estados que mudam lentamente nos quais o sistema persiste por muito tempo), sugerindo que a crise afetou o mercado brasileiro da soja de forma a torná-lo menos eficiente do que era antes. Os índices de RQA foram menores para as séries de preços em dólar, indicando que o mercado externo da soja é mais eficiente que seu mercado interno.

Em trabalho futuro, pode-se analisar outras commodities agrícolas que fazem parte do mercado de exportação brasileiro, como açúcar, milho, carne de boi e frango.

\section{Agradecimentos}

Pelo apoio e incentivo oferecidos para o desenvolvimento deste trabalho, os autores agradecem à Universidade Federal Rural de Pernambuco (UFRPE), ao Programa de Pós-Graduação em Biometria e Estatística Aplicada (PPGBEA), à Coordenação de Aperfeiçoamento de Pessoal de Nível Superior (CAPES), ao Conselho Nacional de Desenvolvimento Científico e Tecnológico (CNPq), e à Fundação de Amparo à Ciência e Tecnologia do Estado de Pernambuco (FACEPE).

\section{Referências}

Barros, V. da S., Santana, L. I. T. de, Silva, J. M. da, Araújo, L. da S., Albuquerque, C. R., \& Stosic, T. (2021). Analysis of time series of hot pixels in brazilian biomes using the Recurrence Plot method. Research, Society and Development, 10(4), e16010413925.

Bastos, J. A., \& Caiado, J. (2011). Recurrence quantification analysis of global stock markets. Physica A: Statistical Mechanics and its Applications, 390(7), $1315-1325$

Cao, L. (1997). Practical method for determining the minimum embedding dimension of a scalar time series. Physica D: Nonlinear Phenomena, 110(1-2), 4350 .

CEPEA/ESALQ/USP - Centro de Estudos Avançados em Economia Aplicada / Escola Superior de Agricultura Luiz de Queiroz / Universidade de São Paulo. (2021). https://www.cepea.esalq.usp.br/br.

CNA - Confederação de Agricultura e Pecuária. (2021). www.cnabrasil.org.br. 
Desai, U., Martis, R. J., Acharya, U. R., Nayak, C. G., Seshikala, G., \& Shetty K, R. A. N. J. A. N. (2016). Diagnosis of multiclass tachycardia beats using recurrence quantification analysis and ensemble classifiers. Journal of Mechanics in Medicine and Biology, 16(01), 1640005.

de Santana, L. I. T., da Silva, J. M., da Silva Araújo, L., Moreira, G. R., \& Stosic, T. (2020). Análise de quantificação de recorrência de preços brasileiros do milho, da soja e da carne de frango. Research, Society and Development, 9(10), e9979109461-e9979109461.

de Santana, L. I. T., de Carvalho Barreto, I. D., da Silva Araújo, L., \& Stosic, T. (2020). Recurrence quantification analysis of São Francisco River flow: hydrological alterations caused by the construction of Sobradinho dam. Research, Society and Development, 9(11), e87491110467-e87491110467.

Donner, R. V., Balasis, G., Stolbova, V., Georgiou, M., Wiedermann, M., \& Kurths, J. (2019). Recurrence-Based Quantification of Dynamical Complexity in the Earth's Magnetosphere at Geospace Storm Timescales. Journal of Geophysical Research: Space Physics, 124(1), 90-108.

Eckmann, J. P., Kamphorst, S. O., \& Ruelle, D. (1987). Recurrence Plots of Dynamical Systems. Europhysics Letters, 4(9), 973-977.

FEALQ - Fundação de Estudos Agrários Luiz de Queiroz. (2021). https://fealq.org.br/.

Goldberg, R. A., \& Davis, J. H. (1957). A concept of agribusiness. Harvard university, Boston.

Gouveia, A. B. V. S., Paulo, L. M. de, Silva, J. M. S. da, Sousa, F. E. de, Santos, F. R. dos, \& Minafra, C. S. (2020). Soybean by-products in bird feeding: Review. Research, Society and Development, 9(7), e471974187.

Ivanic, M., Martin, W., \& Zaman, H. (2012). Estimating the short-run poverty impacts of the 2010-11 surge in food prices. World Development, 40(11), 23022317 .

Kantz, H., \& Schreiber, T. (2004). Nonlinear time series analysis (Vol. 7). Cambridge university press.

Kristoufek, L., Janda, K., \& Zilberman, D. (2012). Correlations between biofuels and related commodities before and during the food crisis: A taxonomy perspective. Energy Economics, 34(5), 1380-1391.

Marwan, N., Romano, M. C., Thiel, M., \& Kurths, J. (2007). Recurrence plots for the analysis of complex systems. Physics reports, 438(5-6), 237-329.

Marwan, N., Wessel, N., Meyerfeldt, U., Schirdewan, A., \& Kurths, J. (2002). Recurrence-plot-based measures of complexity and their application to heartrate-variability data. Physical review E, 66(2), 026702.

Mauad, M., Silva, T. L. B., Neto, A. I. A., \& Abreu, V. G. (2010). Influência da densidade de semeadura sobre características agronômicas na cultura da soja. Agrarian, 3(9), 175-181.

Piccoli, E. (2018). A Importância Da Soja Para O Agronegócio: Uma análise sob o enfoque do aumento da produção de agricultores no Município de Santa Cecília do Sul. FAT-Faculdade e Escola Curso Aapejara/RS. https://www.fatrs.com.br/faculdade/uploads/tcc/d464ec1e2f2c450aa33bb0e990b54878.pdf

R Core Team (2020). R: A language and environment for statistical computing. R Foundation for Statistical Computing, Vienna, Austria. URL https://www.Rproject.org/.

Salomão, R. (2020). Exportações do agronegócio somaram US\$ 96,79 bilhões em 2019. https://revistagloborural.globo.com/No ticias/Economia/noticia/2020/01/exportacoes-do-agronegocio-somaram-us-9679-bilhoes-em-2019.html.

Shu, Z. R., Chan, P. W., Li, Q. S., He, Y. C., \& Yan, B. W. (2021). Investigation of chaotic features of surface wind speeds using recurrence analysis. Journal of Wind Engineering and Industrial Aerodynamics, 210, 104550.

SNA - Sociedade Nacional de Agricultura. (2021). Balanço 2020: commodities que bateram recordes de exportação. www.sna.agr.br.

Takens, F. (1981). Detecting strange attractors in turbulence. In Dynamical systems and turbulence, Warwick 1980 (pp. 366-381). Springer, Berlin, Heidelberg.

Webber Jr, C. L., \& Zbilut, J. P. (2005). Recurrence quantification analysis of nonlinear dynamical systems. Tutorials in contemporary nonlinear methods for the behavioral sciences, 94(2005), 26-94.

Yao, C. Z., \& Lin, Q. W. (2017). Recurrence plots analysis of the CNY exchange markets based on phase space reconstruction. The North American Journal of Economics and Finance, 42, 584-596.

Zbilut, J. P. (2005). Use of recurrence quantification analysis in economic time series. In Economics: Complex Windows (pp. 91-104). Springer, Milano.

Zbilut, J. P., \& Webber Jr, C. L. (1992). Embeddings and delays as derived from quantification of recurrence plots. Physics letters A, 171(3-4), $199-203$. 